\title{
Propriedades Psicométricas da Comprehensive Assessment of Sadistic Tendencies (CAST) no Brasil
}

\author{
Renan P. Monteiro ${ }^{1}$ \\ Emerson Diógenes de Medeiros ${ }^{2}$ \\ Clécia Lino da Silva ${ }^{1}$ \\ Izadora Mendonça de Melo \\ Fabrício Antônio Duarte Figueiredo ${ }^{1}$ \\ Brunna Belmonte Dorileo \\ ${ }^{1}$ Universidade Federal de Mato Grosso, Cuiabá, Mato Grosso, Brasil \\ ${ }^{2}$ Universidade Federal do Delta do Parnaíba, Parnaíba, Piamí, Brasil
}

\begin{abstract}
Resumo
Objetivou-se reunir evidências psicométricas da Comprehensive Assessment of Sadistic Tendencies (CAST) no Brasil. Os resultados da análise fatorial exploratória indicaram uma solução unifatorial, com adequada precisão, tendo itens com discriminação entre moderada e muito alta, cobrindo uma ampla faixa do traço latente. As correlações da CAST com a Tríade Sombria, amabilidade e conscienciosidade e com os fatores da escala de agressão são evidências de validade convergente, além do sadismo ter um incremento significativo na explicação do comportamento agressivo. Pensando no avanço dos estudos sobre a Tétrade Sombria, selecionaram-se os quatro itens mais informativos da CAST, acoplando-os aos 12 da Dirty Dozen, testados por meio de uma análise fatorial confirmatória, apresentaram indicadores de ajuste aceitáveis. No conjunto, os resultados reúnem evidências da adequação psicométrica da CAST, oferecendo uma versão curta e eficaz para avaliação da Tétrade Sombria.

Palavras-chave: sadismo, personalidade, tétrade sombria, validação
\end{abstract}

Psychometric properties of the Comprehensive Assessment of Sadistic Tendencies (CAST) in Brazil

\begin{abstract}
The objective of the study was to collect psychometric evidence for the Comprehensive Assessment of Sadistic Tendencies (CAST) in Brazil. The results of the Exploratory Factor Analysis indicated a unifactorial solution with adequate reliability and moderate to high discrimination items, covering a wide range of the latent trait. Correlations of the CAST with the Dark Triad, agreeableness, conscientiousness, and with the factors of the aggression scale are evidence of convergent validity. Moreover, sadism significantly increases the explanation of aggressive behavior. Bearing in mind the development of studies on the Dark Tetrad, the four most informative items of the CAST combined with the 12 items of the Dirty Dozen were tested using a Confirmatory Factor Analysis and presented acceptable indexes of fit. Overall, the findings gather evidence of psychometric suitability of the CAST and provide a short and efficient tool to assess the Dark Tetrad.
\end{abstract}

Keywords: sadism; personality; Dark Tetrad; validation

Propiedades Psicométricas de la Comprehensive Assessment of Sadistic Tendencies (CAST) en Brasil

\section{Resumen}

Se buscó reunir evidencias psicométricas de la Comprehensive Assessment of Sadistic Tendencies (CAST) en Brasil. Los resultados del Análisis Factorial Exploratorio indicaron una solución unifactorial, con adecuada precisión, teniendo ítems con discriminación moderada y muy alta, cubriendo una amplia parte del rasgo latente. Las correlaciones de la CAST con la Tríada Oscura, amabilidad y concienciosidad y con los factores de la escala de agresión son evidencias de validez convergente, además del sadismo tener un incremento significativo en la explicación del comportamiento agresivo. Considerando el curso de los estudios sobre la Tétrada Oscura, se seleccionaron los cuatro ítems más informativos de la CAST, acoplándolos a los 12 de Dirty Dozen, evaluados por medio de un Análisis Factorial Confirmatorio, presentaron indicadores de ajuste aceptables. En el conjunto, los resultados reúnen evidencias de la adecuación psicométrica de la CAST, ofreciendo una versión corta y eficaz para la evaluación de la Tétrada Oscura.

Palabras clave: sadismo; personalidad; Tétrada Oscura; validación.

\section{Introdução}

O estudo dos traços sombrios da personalidade tem crescido substancialmente nos últimos 15 anos. A importância da temática é constatada tendo em conta produções que sintetizam o conhecimento na área
(Furnham, Richards, \& Paulhus, 2013), Handbooks que discutem o lado obscuro da personalidade (Zeigler-Hill \& Marcus, 2016) e edições especiais de importantes jornais de personalidade (e.g., Personality and Individual Differences) voltados para traços malévolos (Medeiros, Monteiro, Gouveia, Nascimento, \& Gouveia, 2017). 
Nesse contexto, a proposição da Tríade Sombria (Paulhus \& Williams, 2002) foi um marco importante, indicando que o lado sombrio da personalidade é formado por maquiavelismo (e.g., manipulam e retardam gratificações visando ganhos pessoais) e pelas variações subclínicas de psicopatia (e.g., impulsivos, buscadores de sensações e insensíveis aos demais) e narcisismo (e.g., possuem autoestima grandiosa e senso de merecimento). Chabrol, Van Leeuwen, Rodgers e Séjourné (2009) propuseram a extensão desse modelo, incluindo o sadismo nessa constelação do mal, formando a Tétrade Sombria da personalidade.

No contexto brasileiro, os estudos sobre personalidade ainda são tímidos e focam nos Cinco Grandes Fatores (CGF; Laros, Peres, Andrade, \& Passos, 2018). É importante ter em conta modelos que se concentram em traços obscuros que, apesar de indesejáveis, fazem parte de uma faixa normal de funcionamento da personalidade, relacionando-se a desfechos negativos, a exemplo de comportamentos agressivos e de risco no trânsito (Monteiro, Coelho, Hanel, Pimentel, \& Gouveia, 2018), vingança (Coelho et al., 2018) e comportamentos contraproducentes em contexto laboral (Ying \& Cohen, 2018). Apesar da relevância, apenas recentemente foram disponibilizadas medidas adequadas para avaliação da Tríade Sombria em contexto brasileiro (e.g., Gouveia, Monteiro, Gouveia, Athayde, \& Cavalcanti, 2016; Medeiros et al., 2017). O presente estudo busca contribuir com a expansão da temática no Brasil, adaptando uma medida para avaliação de traços sádicos da personalidade (Comprehensive Assessment of Sadistic Tendencies; Buckels, 2018). A seguir o sadismo será conceituado, sendo listado alguns de seus correlatos e instrumentos para a sua mensuração.

\section{Traços Sádicos de Personalidade}

O interesse pelo sadismo como um traço de personalidade é recente em razão das evidências de sua existência em nível subclínico (i.e., distribui-se ao longo de um contínuo; Buckels, 2018; O’Meara, Davies, \& Hammond, 2011). Esse traço é fortemente associado com suas expressões extremas (e.g., contextos sexual e prisional), que são coibidas pela sociedade contemporânea, algo que levou ao desenvolvimento de uma variação branda, conhecida como sadismo cotidiano (everyday sadism; Paulhus \& Dutton, 2016). O sadismo pode ser entendido como a obtenção de prazer ao ver ou fazer sofrer aos demais (Greitemeyer, 2015; Paulhus, 2014), sendo que tais tendências sádicas se expressam de variadas formas, como infringir sofrimento direto a outrem por meio de agressão física ou humilhações verbais. Não obstante, há outras formas de expressá-la de modo que não representem transgressões legais, a exemplo de assistir a conteúdos violentos na televisão ou jogar videogames violentos (Greitemeyer, 2015; Greitemeyer \& Sagioglou, 2017).

Ademais, estudos têm verificado que o sadismo cotidiano tem se relacionado com mau comportamento na internet (e.g., trolagens, cyberbullying; Buckels, Trapnell, Andjelovic, \& Paulhus, 2018; Craker \& March, 2016; Sest \& March, 2017; van Geel, Goemans, Toprak, \& Vedder, 2017), bullying (van Geel et al. 2017), além de predizer a aceitação de mitos sobre o estupro e dominância sexual (Russell, Doan, \& King, 2017). As motivações para dominar e machucar que pessoas com traços sádicos possuem se estende para além das relações interpessoais, de modo que esse traço de personalidade relaciona-se com o vandalismo (i.e., destruir as coisas por prazer; Pfattheicher, Keller, \& Knezevic, 2018). As evidências listadas previamente apontam para a relevância de se estudar o fenômeno, sendo que o sadismo pode representar um importante preditor de atitudes e comportamentos prejudiciais às demais pessoas, a exemplo de violência doméstica e agressão entre pares. Ressalta-se que medidas que operacionalizam o sadismo fomentaram o desenvolvimento de estudos e o conhecimento dos seus correlatos, não obstante, não foi encontrada qualquer medida adaptada para o contexto brasileiro, o que pode representar um entrave para o avanço teórico e empírico desse tema no país.

No que tange a mensuração de traços sádicos, na literatura consultada foi possível identificar quatro instrumentos (Visser \& Campbell, 2018): Short Sadistic Impulse Scale (SSIS; O'Meara et al., 2011), Assessment of Sadistic Personality (ASP; Plouffe, Saklofske, \& Smith, 2017), Varieties of Sadistic Tendencies (VAST; Paulhus \& Jones, 2015) e Comprehensive Assessment of Sadistic Tendencies (CAST; Buckels, 2018). A SSIS foi a primeira medida construída para avaliação da variação subclínica do sadismo, sendo formada por 10 itens que compõem um fator geral. Entretanto, tais itens são muito homogêneos, possuindo baixa validade de conteúdo (Plouffe et al., 2017). Por outro lado, a ASP foi desenhada para avaliar de forma mais abrangente o sadismo, tendo em conta itens que versam sobre subjugação, busca por prazer e falta de empatia. Não obstante, itens que fazem menção à subjugação e falta de empatia não fazem referência direta ao prazer oriundo da crueldade, podendo ser confundido com outras motivações para agredir (Buckels, 2018), além da falta de empatia 
ser um componente comum a outros traços sombrios (Paulhus, 2014). Nessa direção, é importante contar com uma medida que omita aspectos gerais e comuns de diferentes construtos e foque nos aspectos centrais que diferenciam o sadismo de seus correlatos (Buckels, 2018; Paulhus, 2014).

Mesmo pensada para avaliar diferentes dimensões do sadismo, a ASP possui uma estrutura unifatorial (Plouffe, Smith, \& Saklofske, 2018), a exemplo da SSIS, sugerindo que a dimensionalidade do sadismo ainda é obscura. Portanto, é fundamental contar com medidas que considerem, em diferentes contextos, o prazer oriundo da crueldade (Buckels, 2018) que é o aspecto central do sadismo (Paulhus, 2014). Considerando o previamente comentado, a VAST representa um avanço ao considerar variações sutis do sadismo, não se referindo unicamente à agressão direta física, mas também por apresentar uma forma vicária de expressão (e.g., jogar videogames violentos ou assistir a filmes e esportes violentos). Sua versão atualizada, a CAST, foi pensada para expandir o conteúdo da medida cobrindo a expressão verbal do sadismo, assim como sua expressão física e vicária (Buckels, 2018). Por meio de análise fatorial exploratória, Buckels verificou uma estrutura de três fatores, além de reunir indicadores aceitáveis por meio de uma análise fatorial confirmatória para um modelo com um fator geral e três de primeira ordem (e.g., CFI $=0,93$; RMSEA $=0,06$ ), observando coeficientes alfa que variaram entre 0,81 e 0,83 e estabilidade temporal (teste reteste no intervalo de um ano) que variou entre 0,64 e 0,78 .

Em relação à validade convergente, Buckels (2018) observou que a CAST e seus três fatores correlacionaram-se positivamente com a SSIS, com psicopatia, maquiavelismo e narcisismo. A pontuação total da CAST e seus fatores específicos também se correlacionaram com o fator geral do Questionário de Agressão de Buss-Perry e seus quatro fatores específicos (agressão física, agressão verbal, raiva e hostilidade). Por fim, verificou-se que o sadismo associou-se negativamente com dois traços do modelo CGF: amabilidade e conscienciosidade.

Inexistindo estudos sobre sadismo, e as evidências promissoras da CAST, adaptá-la para o Brasil é importante para ensejar futuras pesquisas. Portanto, serão verificadas a estrutura fatorial e a fidedignidade dessa medida, analisando os parâmetros individuais de seus itens e sua validade convergente e incremental. Considerando que os traços sombrios apresentam aspectos em comum, como baixa empatia e o antagonismo (Međedović \& Petrović, 2015; Paulhus, 2014), espera-se que o sadismo se correlacione positivamente com psicopatia, narcisismo e maquiavelismo. Ademais, a baixa amabilidade é um dos aspectos centrais dos traços obscuros, e sádicos não controlam seus impulsos destrutivos para obter prazer vendo ou fazendo os demais sofrerem, sendo hipotetizado que os marcadores amabilidade e conscienciosidade se correlacionarão negativamente com o sadismo (Buckels, 2018). Por fim, agressões físicas e verbais são usadas por sádicos para infringir dor e obter prazer com o sofrimento de outras pessoas. Logo, espera-se que o sadismo explique um incremento significativo do comportamento agressivo (Buckels, 2018; Chester, DeWall, \& Enjaian, 2019).

\section{Método}

\section{Participantes}

Participaram 200 pessoas, com idades entre 18 e $63 \operatorname{anos}(M=26,8 ; D P=9,06)$, em maioria do sexo feminino $(64,5 \%)$, pessoas com ensino superior incompleto $(44,5 \%)$, que se autodeclararam solteiros $(68 \%)$ e de classe social média (46\%).

\section{Instrumentos}

Para a mensuração de traços sádicos da personalidade, foi utilizada a Comprehensive Assessment of Sadistic Tendencies (CAST; Buckels, 2018). Essa medida é formada por 18 itens, sendo os participantes orientados a indicar seu nível de concordância $(1-$ Discordo Fortemente; 7 - Concordo Fortemente) a itens como "Gosto de machucar fisicamente as pessoas" (Sadismo Físico), "Gosto de fazer piadas às custas dos outros" (Sadismo Verbal) e "Gosto de assistir a lutas de ringue (e.g., MMA), nas quais os lutadores não tem por onde escapar" (Sadismo Vicário).

Em relação ao processo de tradução da CAST, esta foi realizada do inglês para o português por dois psicólogos bilíngues com expertise na área de personalidade sombria. Um terceiro psicólogo, também bilíngue e com experiência na área, retraduziu os itens do português para o inglês, comparando as versões e prezando pelo consenso. A versão preliminar da medida foi aplicada em 10 universitários, buscando verificar incompreensões nos itens e escala de resposta. Ressalta-se que desse procedimento não se demandou qualquer alteração no instrumento.

Para estimar a Tríade Sombria, utilizou-se a versão brasileira (Gouveia, Monteiro, Gouveia, Athayde, \& 
Cavalcanti, 2016) do Dirty Dozen (Jonason \& Webster, 2010). Essa medida é formada por 12 itens, quatro por dimensão, sendo os participantes orientados a indicar o seu grau de concordância (1 - Discordo Fortemente; 5 - Concordo Fortemente) a afirmações como "Costumo manipular os outros para conseguir o que quero" (Maquiavelismo), "Costumo ser insensível ou indiferente" (Psicopatia) e "Tendo a querer que os outros me admirem" (Narcisismo).

A versão brasileira (Pimentel, Ferreira, Vargas, Maynart, \& Mendonça, 2014) do Ten-Item Personality Inventory (Gosling, Rentfrow, \& Swann Jr., 2003) foi utilizada para operacionalizar os Cinco Grandes Fatores. Essa medida é formada por 10 itens, sendo dois para cada dimensão da personalidade. Considerando a seguinte frase estímulo "Eu me vejo como alguém...", os participantes indicavam o seu nível de concordância (1 - Discordo Fortemente; 7 - Concordo Fortemente) a itens como "Extrovertido, entusiasta" (Extroversão), "Simpático, acolhedor" (Amabilidade), "Confiável, autodisciplinado" (Conscienciosidade), "Ansioso, que se chateia facilmente" (Neuroticismo) e "Aberto a novas experiências, complexo" (Abertura).

Para mensurar a agressividade, recorreu-se à versão brasileira (Monteiro et al., 2018) da Brief Aggression Questionnaire (BAQ; Webster et al., 2014). Essa medida é composta por 12 itens, distribuídos equitativamente em quatro fatores. Os participantes são orientados a indicar o quanto concordam (1 - Discordo Totalmente; 5 - Concordo Totalmente) com afirmações como "Se uma pessoa me provocar o suficiente, eu posso chegar a agredi-la" (Agressão Física), "Quando as pessoas me irritam, posso chegar a dizer a elas o que penso sobre elas" (Agressão Verbal), "Às vezes me descontrolo sem uma boa razão" (Raiva) e "Às vezes eu sinto que as pessoas estão zombando de mim pelas costas" (Hostilidade).

\section{Procedimento}

Os dados foram coletados por meio de um questionário on-line, construído na plataforma Google Formulários, sendo o link compartilhado nas redes sociais. Prévio a apresentação das medidas, descreveu-se os objetivos do estudo, além de ter sido destacado o caráter anônimo e confidencial das respostas, a natureza voluntária da participação, podendo o participante desistir a qualquer momento sem acarretar ônus. Posteriormente, demandava-se que os partícipes expressassem a sua concordância em participar do estudo, marcando uma opção correspondente ao final do Termo de Consentimento Livre e Esclarecido, seguindo os preceitos éticos para pesquisas em ciências humanas e sociais (Resolução 510/16).

\section{Análise de Dados}

Os dados foram analisados por meio do SPSS, Factor (Lorenzo-Seva \& Ferrando, 2006) e R (R Development Core Team, 2015). O primeiro foi utilizado para calcular estatísticas descritivas (caracterizar a amostra), além de se calcular as correlações entre as variáveis (validade convergente) e regressão hierárquica (validade incremental). O Factor foi empregado para explorar a estrutura fatorial da CAST, além de se calcular os coeficientes de consistência interna (alfa de Cronbach e ômega de McDonald). Por fim, o R, especificamente o pacote MIRT (Chalmers, 2012), foi utilizado para calcular os parâmetros individuais dos itens (dificuldade e discriminação), utilizando o Modelo de Resposta Graduada (Samejima, 1969). Ainda com o R, mas utilizando os pacotes Lavaan (Rosseel, 2012) e SemPlot (Epskamp, 2017), realizou-se uma análise fatorial confirmatória verificando o ajuste de uma versão concisa para operacionalização da Tétrade Sombria, tendo em conta os seguintes indicadores de ajuste aos dados: Razão Qui-Quadrado por graus de liberdade $\left(\chi^{2} / g \emptyset\right)$, Comparative Fit Index (CFI), Goodness of Fit Index (GFI), Adjusted Goodness of Fit Index (AGFI), Tucker-Lewis Index (TLI) e Root Mean-Square Error of Approximation (RMSEA). Para ser considerado aceitável, o modelo deve ter valores de CFI, GFI, AGFI e TLI acima de 0,90, de preferência acima de 0,95, e o RMSEA abaixo de 0,08 (Brown, 2006; Kline, 2016).

\section{Resultados}

Inicialmente, verificou-se que o Kaiser-Meyer-Olkin $(0,85)$ e o Teste de Esfericidade de Bartlett $\left[\chi^{2}\right.$ $=1248$ (153), $p<0,001]$ indicaram a possibilidade de se realizar uma análise fatorial exploratória, utilizando matriz de correlações policóricas e estimador não paramétrico (Unweighted Least Squares; ULS). Os resultados indicaram uma solução com quatro fatores com autovalores maiores que um $(8,25 ; 1,34 ; 1,16 ; 1,03)$, não obstante, o critério de Kaiser tende a superestimar o número de fatores (Laros, 2012). Nessa direção, recorreu-se ao critério de Hull que, em estudos de simulação, tem se mostrado como o mais preciso na retenção de fatores (Lorenzo-Seva, Timmerman, \& Kiers, 2011), sendo que, na presente ocasião, tal critério indicou uma solução unifatorial como a mais parcimoniosa, 
explicando $46 \%$ da variância total, sendo possível observar as cargas fatoriais na Tabela 1.

Considerando a magnitude das cargas fatoriais, foram excluídos dois itens com saturações abaixo do ponto de corte estabelecido $|0,30|$ (Item 15. Eu jamais humilharia alguém de propósito; Item 18. Esportes são violentos demais). Os dezesseis restantes apresentaram cargas fatoriais entre 0,58 [Item 9. Gosto de assistir lutas de ringue (e.g., MMA), nas quais os lutadores não tem como escapar] a 0,79 (Item 14. Talvez eu não deveria, mas nunca me canso de zombar de alguns colegas), tendo o instrumento adequada consistência interna, avaliada por meio do ômega de McDonald e alfa de Cronbach $(\omega=0,92$ e $\alpha=0,92)$.

O passo seguinte foi estimar os parâmetros individuais dos 16 itens (Tabela 1), especificamente a discriminação (parâmetro "a"), dificuldade (parâmetro "b") e informação psicométrica. A discriminação se refere a capacidade do item em diferenciar pessoas ao longo do traço latente (sadismo), sendo possível observar que sete itens apresentaram discriminação moderada ("a" entre 0,65 e 1,34), quatro apresentaram discriminação alta ("a" entre 1,35 a 1,69) e cinco apresentaram discriminação muito alta ("a" $>$ 1,70; Baker, 2001). Em relação ao parâmetro de dificuldade, que indica o nível de traço latente que o indivíduo precisa ter para endossar a opção de resposta, observa-se que os itens abrangem grande porção do traço latente, variando entre $-0,30$ a 4,16. Inspecionando a informação psicométrica da escala (Figura 1), verifica-se que o teste é mais preciso na avaliação de pessoas com níveis moderados e acentuados de sadismo, avaliando inadequadamente a porção inferior do traço, sendo os itens 14, 8, 12 e 7 os mais informativos.

Observa-se que a versão de 16 itens da CAST se correlacionou com os traços de maquiavelismo $(r$ $=0,44, p<0,001)$, psicopatia $(r=0,51, p<0,001) \mathrm{e}$ narcisismo $(r=0,39, p<0,001)$. Tendo em conta os fatores do CGF, verificou-se que a pontuação total da CAST se correlacionou com amabilidade $(r=-0,19$, $p<0,01)$ e conscienciosidade $(r=-0,30, p<0,001)$. Por fim, o fator geral da CAST se correlacionou com agressão física $(r=0,52, p<0,001)$, agressão verbal $(r$ $=0,34, p<0,001)$, raiva $(r=0,21, p<0,001)$ e hostilidade $(r=0,30, p<0,001)$.

Tabela 1.

Cargas Fatoriais e Parâmetros Individuais dos Itens

\begin{tabular}{cccccccccc}
\hline & Fator & $b^{2}$ & $\mathrm{a}$ & $\mathrm{b}_{1}$ & $\mathrm{~b}_{2}$ & $\mathrm{~b}_{3}$ & $\mathrm{~b}_{4}$ & $\mathrm{~b}_{5}$ & $\mathrm{~b}_{6}$ \\
\hline Item 14 & 0,79 & 0,62 & 2,79 & 0,41 & 0,91 & 1,09 & 1,53 & 2,11 & - \\
Item 2 & 0,77 & 0,60 & 1,43 & 1,57 & 2,65 & 2,86 & 3,37 & 4,00 & - \\
Item 11 & 0,76 & 0,57 & 1,66 & 0,71 & 1,16 & 1,41 & 1,96 & 2,34 & 2,69 \\
Item 8 & 0,75 & 0,56 & 2,33 & 0,30 & 0,92 & 1,27 & 1,43 & 1,81 & 1,97 \\
Item 4 & 0,74 & 0,55 & 1,41 & 1,30 & 1,73 & 2,20 & 2,60 & 3,30 & 3,71 \\
Item 13 & 0,71 & 0,51 & 1,13 & 1,92 & 2,42 & 2,61 & 2,92 & 3,50 & 3,84 \\
Item 1 & 0,71 & 0,50 & 1,38 & 0,66 & 1,43 & 1,80 & 2,68 & 3,34 & 4,16 \\
Item 10 & 0,70 & 0,48 & 1,29 & 1,12 & 1,59 & 1,90 & 2,25 & 2,89 & 3,46 \\
Item 17 & 0,69 & 0,48 & 1,26 & 0,91 & 1,57 & 1,99 & 2,56 & 2,97 & 3,79 \\
Item 12 & 0,69 & 0,47 & 2,01 & 0,22 & 0,76 & 1,07 & 1,55 & 2,35 & 2,81 \\
Item 5 & 0,64 & 0,41 & 1,78 & $-0,10$ & 0,59 & 0,94 & 1,20 & 2,00 & 2,53 \\
Item 16 & 0,63 & 0,40 & 0,94 & 0,98 & 2,08 & 2,82 & 3,79 & 4,59 & - \\
Item 6 & 0,63 & 0,40 & 1,10 & 0,70 & 1,28 & 1,57 & 2,00 & 2,52 & 2,93 \\
Item 3 & 0,62 & 0,39 & 1,33 & $-0,01$ & 0,80 & 1,28 & 1,71 & 2,31 & 2,84 \\
Item 7 & 0,62 & 0,39 & 1,81 & $-0,30$ & 0,34 & 0,76 & 1,12 & 1,73 & 2,14 \\
Item 9 & 0,58 & 0,34 & 0,92 & 0,52 & 1,29 & 1,53 & 2,07 & 2,91 & 3,42 \\
Item 15 & 0,22 & 0,05 & - & - & - & - & - & - & - \\
Item 18 & -0.10 & 0,01 & - & - & - & - & - & - & - \\
\hline
\end{tabular}



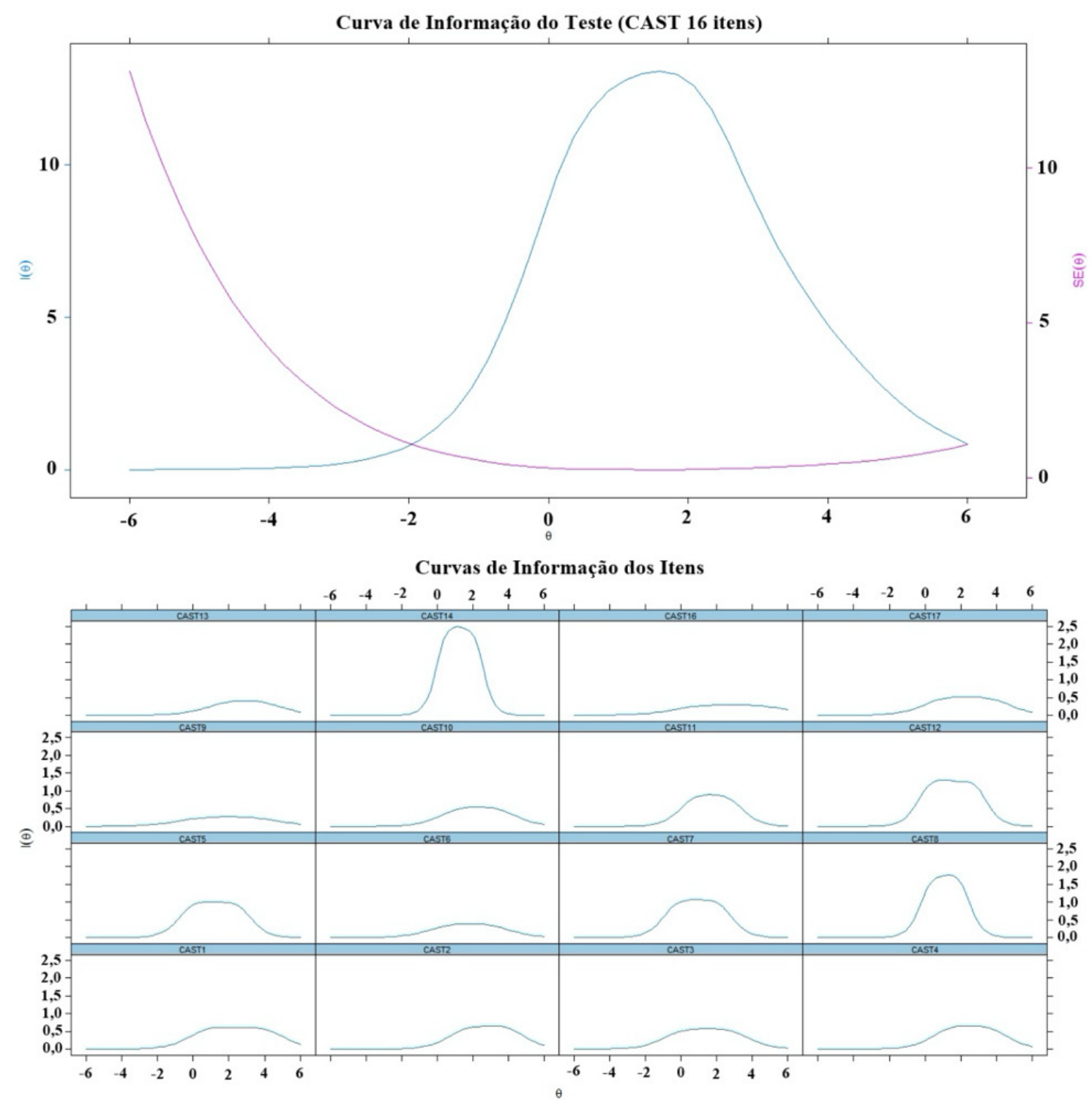

Figura 1. Curvas de informação do Teste e dos itens da CAST.

Duas análises de regressão hierárquica foram realizadas para se verificar a validade incremental da CAST. $\mathrm{Na}$ primeira análise, teve-se como critério a agressão física, entrando no primeiro passo da análise com os traços que formam os CGF, no segundo passo com a Tríade Sombria e no terceiro passo com a pontuação total da CAST. No primeiro passo da análise, os CGF explicaram $6 \%$ da variabilidade na agressão física. Com a inclusão da Tríade Sombria no segundo passo da análise, a variância explicada passou para 19\%, sendo esse incremente de $13 \%$ estatisticamente significativo $(p<$ $0,001)$. Por fim, ao incluir no terceiro passo da análise a pontuação total da CAST, verificou-se que a variância explicada passou para 30\%, incremento de 11\% $(p<$ $0,001)$. No modelo final, apenas o sadismo permaneceu como preditor estatisticamente significativo $(\beta=0,43$; $p<0,001)$. Na segunda análise, a variável critério foi a agressão verbal, seguindo os mesmos passos descritos anteriormente. No primeiro passo, os CGF explicaram $11 \%$ da variabilidade da agressão verbal. Com a inclusão da Tríade Sombria no segundo passo da análise, a variância explicada passou para 19\%, sendo esse incremente de $8 \%$ estatisticamente significativo $(\phi<$ $0,001)$. Por fim, ao incluir no terceiro passo da análise a pontuação total da CAST, verificou-se que a variância explicada passou para $22 \%$, sendo esse incremento 
de $3 \%(p<0,01)$. No modelo final, os preditores que permaneceram estatisticamente significativos foram abertura $(\beta=0,25 ; p<0,001)$, narcisismo $(\beta=0,19 ; p<$ $0,01)$ e sadismo $(\beta=0,22 ; p<0,001)$.

Finalmente, selecionaram-se os quatro itens mais informativos da CAST (Figura 1) e testou-se o ajuste destes para operacionalizar a Tétrade Sombria, junto com os 12 itens que formam a Dirty Dozen. Para tanto, realizou-se uma análise fatorial confirmatória (estimador Maximum Likelibood Robust; MLR), verificando o ajuste do modelo teórico aos dados $\left(\chi^{2} / g l=2,06\right.$; CFI $=0,92 ; \mathrm{GFI}=0,94 ; \mathrm{AGFI}=0,91 ; \mathrm{TLI}=0,90 ;$ RMSEA $=0,07)$, tendo todos os itens apresentado saturações estatisticamente significativas e diferentes de zero $(\lambda \neq$ $0 ; z>1,96, p<0,05$; Figura 2).

\section{Discussão}

A presente pesquisa teve como objetivo adaptar para o contexto brasileiro a Comprehensive Assessment of Sadistic Tendencies (CAST; Buckels, 2018), explorando sua estrutura fatorial, validade convergente, incremental e parâmetros individuais de seus itens por meio da Teoria de Resposta ao Item. Procedeu-se com a adaptação de tal instrumento por conta da escassez de estudos brasileiros sobre traços socialmente aversivos da personalidade e, em específico, pela ausência de estudos empíricos sobre o sadismo, que é uma variável-chave para o entendimento de uma série de atitudes e comportamentos socialmente desviantes (Pfattheicher et al., 2018; Russell et al., 2017; van Geel et al., 2017).

Em relação à estrutura fatorial, contrapondo o que sugere Buckels (2018), foi encontrado suporte para a estrutura unifatorial, utilizando-se um método robusto e mais preciso para retenção de fatores (Lorenzo-Seva et al., 2011) do que o usado no estudo original que propõe a escala. A literatura tem apontado que o prazer oriundo da crueldade é o aspecto central do sadismo (Paulhus, 2014; Paulhus \& Dutton, 2016), diferenciando-o de construtos correlatos, o que explicaria a interrelação entre os itens da CAST, já que possuem um componente subjacente em comum, justificando a plausibilidade da estrutura unifatorial. Nessa direção, os três fatores propostos por Buckels não se referem propriamente a características psicológicas que desenhariam um perfil sádico, mas sim a obtenção de prazer ao praticar ou ver sofrimento dos demais em diferentes contextos, variando em relação a sua intensidade. A propósito, verifica-se que o padrão de correlações que os três fatores propostos inicialmente por Buckels apresenta com a pontuação total da SSIS e da ASP é similar em magnitude (Buckels, 2018; Plouffe et al., 2018), além de também o ser com variáveis externas (e.g., agressão; Buckels, 2018), ademais a pontuação total da CAST tem

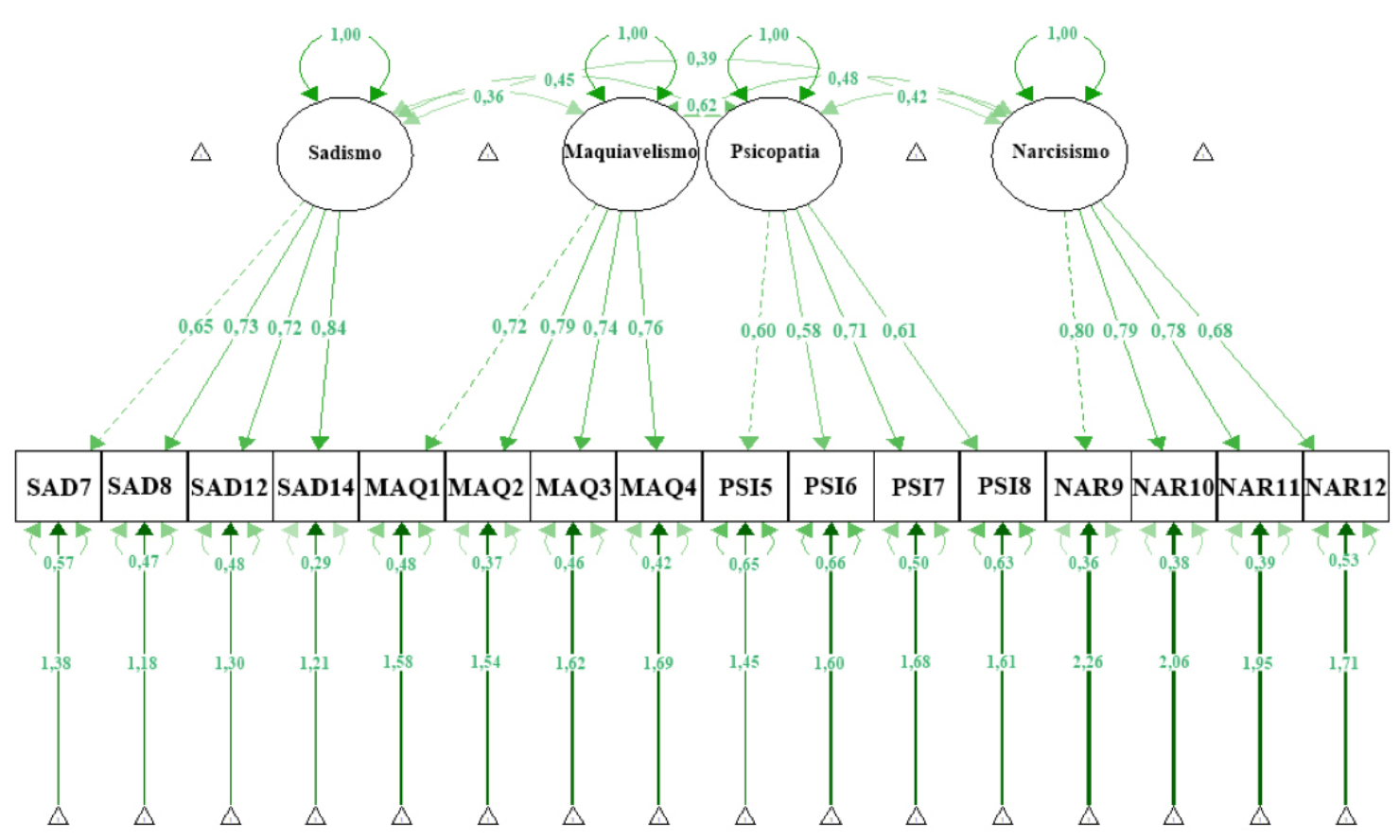

Figura 2. Estrutura fatorial da Tétrade Sombria. 
sido tida em conta em alguns estudos empíricos (Buckels et al., 2018; Gonzalez \& Greitemeyer, 2018).

Tendo em vista o conjunto de evidências reportadas e considerando que apenas recentemente o sadismo subclínico passou a ser mensurado e estudado como um traço, sua dimensionalidade ainda parece pouco clara, indicando ser mais prudente ter em conta um fator geral de sadismo, prática que tem sido recorrente ao se operacionalizar o construto por meio de diferentes medidas (e.g., O’Meara, 2011; Plouffe et al., 2018). No que tange à consistência interna da escala, aferidas por meio do alfa de Cronbach e ômega de McDonald, esta pode ser considerada adequada, já que se encontra acima de pontos de corte sugeridos na literatura (Kline, 2016; Urbina, 2007), além de tais coeficientes reforçarem a razoabilidade da estrutura unifatorial encontrada.

Explorar medidas de sadismo (SSIS, ASP, VAST e CAST) por meio da TRI ainda era uma lacuna na literatura, portanto, o presente estudo representa um avanço empírico nessa direção. A propósito, foi possível verificar que os itens da versão brasileira da CAST conseguem discriminar satisfatoriamente os participantes em função da magnitude de sadismo que apresentam (Baker, 2001). A escala foi desenhada para cobrir diferentes formas de expressar o sadismo, desde formas mais brandas e corriqueiras até aquelas que demandam maior nível de traço latente para serem endossadas. No caso, observou-se que a escala cobre uma amplitude grande do traço latente, contudo, no geral os itens são de difícil endosso por conta da natureza aversiva do traço (Buckels et al., 2018). A TRI possibilitou observar que a versão brasileira da CAST não é precisa para avaliar pessoas na porção inferior do traço, sendo mais adequada para aqueles que possuem nível moderado e alto de sadismo.

Além das evidências sobre a estrutura fatorial, consistência interna e parâmetros dos itens, reuniu-se evidências de validade convergente e incremental. Especificamente, verificou-se que a pontuação total da CAST se correlacionou na direção esperada com os traços que formam a Tríade Sombria, em linha com evidências prévias (Buckels, 2018; Plouffe et al., 2018) que apontam que a relação entre os traços pode ser em função de aspectos como a baixa empatia ou baixa honestidade-humildade (Book et al., 2016; Paulhus, 2014). Foram selecionados os itens mais informativos da CAST para que possam ser utilizados em conjunto com os 12 que formam a Dirty Dozen (Jonason \& Webster, 2010), verificando-se índices de ajuste que atestam para a adequação do modelo teórico aos dados (Brown,
2006; Kline, 2016), dando suporte para o modelo da Tétrade Sombria (Chabrol et al., 2009; Međedović \& Petrović, 2015), possibilitando uma rápida e eficaz avaliação conjunta dos quatro traços em pesquisas com múltiplas variáveis.

Verificou-se, ainda, as relações da CAST com o modelo CGF, sendo que os resultados encontrados seguem o mesmo padrão dos descritos por Buckels (2018). À luz desse modelo geral de personalidade, o sadismo é entendido como a confluência de baixos escores em amabilidade e conscienciosidade. $\mathrm{O}$ antagonismo (baixa amabilidade) é um dos aspectos comuns da Tétrade Sombria, predizendo-os individualmente (Book et al., 2016; Jakobwitz \& Egan, 2006; Paulhus \& Williams, 2002), além de pessoas com tendências sádicas não planejarem suas ações e não terem a capacidade de controlar os seus impulsos destrutivos (baixa conscienciosidade). Por fim, tendo em conta que o sadismo envolve a obtenção de prazer infringindo dor e sofrimento a outras pessoas, são consistentes as relações desse traço com medidas de agressão (Buckels, 2018; Chester et al., 2019). Removendo a variância compartilhada com outros traços de personalidade que compõem a Tríade Sombria e os CGF, o sadismo ocasiona um incremento significativo para a explicação do comportamento agressivo (agressão física e verbal). Agredir envolve a intencionalidade de causar dano no alvo (Anderson \& Huesmann, 2003), sendo que narcisismo prediz agressão em resposta a uma ameaça ao ego e psicopatia o faz mediante provocação sofrida (Jones \& Paulhus, 2010). Sádicos praticam agressão proativa e reativa, percebendo o comportamento agressivo como uma forma de melhorar o humor, sentindo prazer durante o ato agressivo, sobretudo se percebem que a vítima está sofrendo (Chester et al., 2019). O sadismo também prediz o bullying (van Geel et al., 2017), que é um subtipo de comportamento agressivo marcado pela intencionalidade de causar dano na vítima (Medeiros et al., 2015), o que acaba gerando prazer nos sádicos. Portanto, a explicação incremental reside no aspecto único que caracteriza o sadismo, que é a obtenção de prazer por meio da crueldade (Paulhus, 2014).

Apesar das evidências consistentes, os resultados devem ser analisados com cautela. A amostragem não probabilística inviabiliza a generalização dos resultados para além da amostra utilizada. Destaca-se, também, que a avaliação de traços socialmente aversivos pode ser influenciada pela desejabilidade social e que a versão brasileira da CAST não apresenta itens invertidos, o que pode dificultar o controle da 
aquiescência (Gouveia, Mendes, Soares, Monteiro, \& Santos, 2015). Em possibilidades futuras é importante contar com amostras maiores e mais heterogêneas, além de se pensar na possibilidade de se construir uma medida implícita (Gouveia, Athayde, Mendes, \& Freire, 2014) ou mesmo neutralizada para estimação de traços sádicos de personalidade (Costa \& Hauck Filho, 2017). Ademais, é importante proceder com a adaptação das outras medidas de sadismo fazendo comparações diretas com a CAST.

Espera-se que, ao disponibilizar uma medida psicometricamente adequada para o contexto brasileiro, o presente estudo fomente pesquisas sobre sadismo e a Tétrade Sombria. Considerando que o sadismo é um preditor consistente de comportamentos cruéis, ele pode ser uma variável psicológica importante para se compreender e predizer desfechos negativos comuns no contexto brasileiro, como pode ser o caso do bullying, da violência doméstica e do assédio moral em contexto laboral.

\section{Referências}

Anderson, C. A., \& Huesmann, L. R. (2003). Human aggression: A social-cognitive view. Em M. A. Hogg \& J. Cooper (Eds.), Handbook of Social Psychology (pp. 296-323). London: Sage Publications.

Baker, F. B. (2001). The basics of item response theory. Washington, DC: ERIC Clearinghouse on Assessment and Evaluation.

Book, A., Visser, B. A., Blais, J., Hosker-Field, A., Methot-Jones, T., Gauthier, N. Y., ... \& D'Agata, M. T. (2016). Unpacking more "evil": What is at the core of the dark tetrad? Personality and Individual Differences, 90, 269-272. doi: 10.1016/j.paid.2015.11.009

Brown, T. A. (2006). Confirmatory factor analysis for applied research. New York: The Guilford Press.

Buckels, E. E. (2018). The psychology of everyday sadism (Tese de doutorado). The University of British Columbia, Vancouver, Canadá. Recuperado de https://open.library.ubc.ca/cIRcle/collections/ ubctheses/24/items/1.0369056

Buckels, E. E., Trapnell, P. D., Andjelovic, T., \& Paulhus, D. L. (2018). Internet Trolling and Everyday Sadism: Parallel Effects on Pain Perception and Moral Judgment. Journal of Personality, 1-13. doi: 10.1111/jopy.12393
Chabrol, H., Van Leeuwen, N., Rodgers, R., \& Séjourné, N. (2009). Contributions of psychopathic, narcissistic, machiavellian, and sadistic personality traits to juvenile delinquency. Personality and Individual Differences, 47, 734-739. doi: 10.1016/j. paid.2009.06.020

Chalmers, R. P. (2012). MIRT: A multidimensional item response theory package for the $\mathrm{R}$ environment. Journal of Statistical Software, 48, 1-29. Recuperado de https://www.jstatsoft.org/article/view/ v048i06

Chester, D. S., DeWall, C. N., \& Enjaiain, B. (2019). Sadism and Aggressive Behavior: Inflicting Pain to Feel Pleasure. Personality and Social Psychology Bulletin, 45, 1252-1268. doi: 10.1177/0146167218816327

Coelho, G. L., Monteiro, R. P., Hanel, P. H., Vilar, R., Gouveia, V. V., \& Maio, G. R. (2018). Psychometric parameters of an abbreviated vengeance scale across two countries. Personality and Individual Differences, 120,185-192. doi: 10.1016/j.paid.2017.08.042

Costa, A. R. L., \& Hauck Filho, N. (2017). Menos desejabilidade social é mais desejável: Neutralização de instrumentos avaliativos de personalidade. Interaşão em Psicologia, 21, 239-249. doi:10.5380/psi. v21i3.53054

Craker, N., \& March, E. (2016). The dark side of Facebook ${ }^{\circledR}$ : The Dark Tetrad, negative social potency, and trolling behaviours. Personality and Individual Differences, 102, 79-84. doi: 10.1016/j.paid.2016.06.043

Epskamp, S. (2017). Package 'semPlot.' Recuperado de https://cran.r-project. org/web/packages/semPlot/semPlot.pdf

Furnham, A., Richards, S. C., \& Paulhus, D. L. (2013). The Dark Triad of personality: A 10 year review. Social and Personality Psychology Compass, 7, 199-216. doi: $10.1111 / \operatorname{spc} 3.12018$

Gonzalez, J. M., \& Greitemeyer, T. (2018). The relationship between everyday sadism, violent video game play, and fascination with weapons. Personality and Individual Differences, 124, 51-53. doi: 10.1016/j. paid.2017.11.045

Gosling, S. D., Rentfrow, P. J., \& Swann Jr, W. B. (2003). A very brief measure of the Big-Five personality domains. Journal of Research in Personality, 37, 504528. doi: 10.1016/S0092-6566(03)00046-1 
Gouveia, V. V., Athayde, R. A., Mendes, L. A. C., \& Freire, S. E. (2014). Introdução às medidas implícitas: Conceitos, técnicas e contribuições. Diaphora, 12, 80-92. Recuperado de http://www.sprgs.org. br/diaphora/ojs/index.php/diaphora/article/ view $/ 50 / 50$

Gouveia, V. V., Mendes, L. A. C., Soares, A. K. S., Monteiro, R. P., \& Santos, L. C. O. (2015). Escala de Necessidade de Cognição (NCS-18): Efeito de Itens Negativos em sua Estrutura Fatorial. Psicologia: Reflexão e Crítica, 28, 425-433. doi: 10.1590/1678-7153.201528301

Gouveia, V. V., Monteiro, R. P., Gouveia, R. S. V., Athayde, R. A. A., \& Cavalcanti, T. M. (2016). Assessing the dark side of personality: Psychometric evidences of the dark triad dirty dozen. Interamerican Journal of Psychology, 50, 420-432. Recuperado de https://journal.sipsych.org/index.php/IJP/ article/view/126/pdf

Greitemeyer, T. (2015). Everyday sadism predicts violent video game preferences. Personality and Individual Differences, 75, 19-23. doi: 10.1016/j. paid.2014.10.049

Greitemeyer, T., \& Sagioglou, C. (2017). The longitudinal relationship between everyday sadism and the amount of violent video game play. Personality and Individual Differences, 104, 238-242. doi: 10.1016/j. paid.2016.08.021

Jakobwitz, S., \& Egan, V. (2006). The dark triad and normal personality traits. Personality and Individual Differences, 40, 331-339. doi: 10.1016/j. paid.2005.07.006

Jonason, P. K., \& Webster, G. D. (2010). The dirty dozen: A concise measure of the dark triad. Psychological Assessment, 22,420-432. doi: 10.1037/a0019265

Jones, D. N., \& Paulhus, D. L. (2010). Different provocations trigger aggression in narcissists and psychopaths. Social Psychological and Personality Science, 1, 12-18. doi: 10.1177/1948550609347591

Kline, R. B. (2016). Principles and practice of structural equation modeling (4th ed.). New York: Guilford Press.

Laros, J. A. (2012). O uso da Análise Fatorial: Algumas diretrizes para pesquisadores. Em L. Pasquali (Ed.), Análise Fatorial para Pesquisadores (pp. 141-160). Brasília: LABPAM.
Laros, J. A., de Peres, A. J. S., de Andrade, J. M., \& Passos, M. F. D. (2018). Validity evidence of two short scales measuring the Big Five personality factors. Psicologia: Reflexão e Crítica, 31,1-10. doi: 10.1186/ s41155-018-0111-2

Lorenzo-Seva, U., \& Ferrando, P. J. (2006). FACTOR: A computer program to fit the exploratory factor analysis model. Behavior Research Methods, 38, 88-91. doi: 10.3758/BF03192753

Lorenzo-Seva, U., Timmerman, M. E., \& Kiers, H. A. (2011). The Hull method for selecting the number of common factors. Multivariate Behavioral Research, 46, 340-364.doi: 10.1080/00273171.2011.564527

Međedović, J., \& Petrović, B. (2015). The Dark Tetrad. Journal of Individual Differences.

Medeiros, E. D. D., Gouveia, V. V., Monteiro, R. P., Silva, P. G. N. D., Lopes, B. D. J., Medeiros, P. C. B. D., \& Silva, É. S. D. (2015). Escala de Comportamentos de Bullying (ECB): Elaboração e evidências psicométricas. Psico-USF, 20, 385-397. doi: 10.1590/1413-82712015200302

Medeiros, E. D., Monteiro, R. P., Gouveia, R. S. V., Nascimento, B. S., \& Gouveia, V. V. (2017). Dark Triad Dirty Dozen: Avaliando seus Parâmetros Via TRI. Psico-USF, 22, 299-308. doi: 10.1590/1413-82712017220209

Monteiro, R. P., Coelho, G. L. H., Hanel, P. H., Pimentel, C. E., \& Gouveia, V. V. (2018). Personality, dangerous driving, and involvement in accidents: Testing a contextual mediated model. Transportation Research Part F: Traffic Psychology and Behaviour, 58, 106-114. doi: 10.1016/j.trf.2018.06.009

O'meara, A., Davies, J., \& Hammond, S. (2011). The psychometric properties and utility of the Short Sadistic Impulse Scale (SSIS). Psychological Assessment, 23, 523-531. doi: 10.1037/a0022400

Paulhus, D. L. (2014). Toward a taxonomy of dark personalities. Current Directions in Psychological Science, 23(6), 421-426.doi: 10.1177/0963721414547737

Paulhus, D. L., \& Dutton, D. G. (2016). Everyday Sadism. Em V. Zeigler-Hill \& D. K. Marcus (Eds.), The Dark Side of Personality: Science and Practice in Social, Personality, and Clinical Psychology (pp. 109120). Washington, DC: American Psychological Association. 
Paulhus, D. L., \& Jones, D. N. (2015). Measuring dark personalities via questionnaire. Em G. J. Boyle, D. H. Saklofske \& G. Matthews (Eds.), Measures of personality and social psychological constructs (pp.562-594). San Diego, CA: Academic Press.

Paulhus, D. L., \& Williams, K. M. (2002). The Dark Triad of personality: Narcissism, Machiavellianism, and psychopathy. Journal of Research in Personality, 36, 556-563. doi: 10.1016/S0092-6566(02)00505-6

Pfattheicher, S., Keller, J., \& Knezevic, G. (2018). Destroying things for pleasure: On the relation of sadism and vandalism. Personality and Individual Differences. doi: 10.1016/j.paid.2018.03.049

Plouffe, R. A., Saklofske, D. H., \& Smith, M. M. (2017). The assessment of sadistic personality: Preliminary psychometric evidence for a new measure. Personality and Individual Differences, 104, 166-171. doi: 10.1016/j.paid.2016.07.043

Plouffe, R. A., Smith, M. M., \& Saklofske, D. H. (2018). A psychometric investigation of the Assessment of Sadistic Personality. Personality and Individual Differences. doi: 10.1016/j.paid.2018.01.002

Pimentel, C. E., Ferreira, D. C. S., Vargas, M. M., Maynart, V. A. P., \& Mendonça, D. C. (2014). Preferência por estilos de filmes e suas diferenças nos cinco fatores de personalidade. Pesquisas e Práticas Psicossociais, 9, 232-244. Recuperado de http://pepsic. bvsalud.org/pdf/ppp/v9n2/09.pdf

R Development Core Team. (2015). R: A language and environment for statistical computing. Recuperado de https://cran.rproject.org/doc/manuals/fullrefman.pdf

Rosseel, Y. (2012). Lavaan: An R package for structural equation modeling and more. Version 0.5-12 (BETA). Journal of Statistical Software, 48, 1-36. Recuperado de http://users.ugent.be/ yrosseel/ lavaan/lavaanIntroduction.pdf

Russell, T. D., Doan, C. M., \& King, A. R. (2017). Sexually violent women: The PID-5, everyday sadism, and adversarial sexual attitudes predict female sexual aggression and coercion against male victims. Personality and Individual Differences, 111, 242-249. doi: 10.1016/j.paid.2017.02.019
Samejima, F. (1969). Estimation of latent ability using a response pattern of graded scores. Psychometrika Monograph Supplement No. 17. Richmond, VA: Psychometric Society.

Sest, N., \& March, E. (2017). Constructing the cybertroll: Psychopathy, sadism, and empathy. Personality and Individual Differences, 119, 69-72. doi: 10.1016/j. paid.2017.06.038

Urbina, S. (2007). Fundamentos da testagem psicológica. Porto Alegre: Artmed.

van Geel, M., Goemans, A., Toprak, F., \& Vedder, P. (2017). Which personality traits are related to traditional bullying and cyberbullying? A study with the Big Five, Dark Triad and sadism. Personality and Individual Differences, 106, 231-235. doi: 10.1016/j. paid.2016.10.063

Visser, B. A., \& Campbell, S. (2018). Measuring the dark side of personality. Em V. Zeigler-Hill \& T. K. Shackelford (Eds.), Sage Handbook of Personality and Individual Differences (pp.573-591). Londres: SAGE Publications Ltd.

Webster, G. D., DeWall, C. N., Pond, R. S., Deckman, T., Jonason, P. K., Le, B. M., ... \& Smith, C. V. (2014). The brief aggression questionnaire: Psychometric and behavioral evidence for an efficient measure of trait aggression. Aggressive Behavior, 40, 120-139. doi: $10.1002 / a b .21507$

Ying, L., \& Cohen, A. (2018). Dark triad personalities and counterproductive work behaviors among physicians in China. The International Journal of Health Planning and Management, 1-14.doi: 10.1002/ hpm.2577

Zeigler-Hill, V., \& Marcus, D. K. (2016). Introduction: A bright future for dark personality features? Em V. Zeigler-Hill, \& D. K. Marcus (Eds.), The dark side of personality: Science and practice in social, personality, and clinical psychology (pp. 3-22). Washington, DC: American Psychological Association.

Recebido em: 07/12/2018

Reformulado em: 11/12/2019

Aprovado em: 21/01/2020 
Sobre os autores:

Renan P. Monteiro é doutor em Psicologia Social pela Universidade Federal da Paraíba e professor do Departamento de Psicologia da Universidade Federal de Mato Grosso.

ORCID: https://orcid.org/0000-0002-5745-3751

E-mail: renanpmonteiro@gmail.com

Emerson Diógenes de Medeiros é doutor em Psicologia Social pela Universidade Federal da Paraíba e professor do Departamento de Psicologia da Universidade Federal do Delta do Parnaíba.

ORCID: https://orcid.org/0000-0002-1407-3433

E-mail: emersondiogenes@gmail.com

Clécia Lino da Silva é graduanda em Psicologia na Universidade Federal de Mato Grosso.

ORCID: https://orcid.org/0000-0002-7268-9711

E-mail:clecia_lino@hotmail.com

Izadora Mendonça de Melo é graduanda em Psicologia na Universidade Federal de Mato Grosso.

ORCID: https://orcid.org/0000-0002-4587-863X

E-mail:izadora.melo@hotmail.com

Fabrício Antônio Duarte Figueiredo é graduando em Psicologia na Universidade Federal de Mato Grosso.

ORCID: https://orcid.org/0000-0001-6321-1751

E-mail: efeantonio@gmail.com

Brunna Belmonte Dorileo é graduanda em Psicologia na Universidade Federal de Mato Grosso.

ORCID: https://orcid.org/0000-0001-8505-7521

E-mail: brunnadorileo@gmail.com

Contato com os autores:

Renan P. Monteiro

Universidade Federal de Mato Grosso, Campus de Cuiabá, Departamento de Psicologia

Av. Fernando Corrêa da Costa, 2367, Boa Esperança

Cuiabá-MT, Brasil

CEP: 78060-900 